\section{ORIGINAL RESEARCH}

Y.-C. Zhu

C. Dufouil

B. Mazoyer

A. Soumaré

F. Ricolfi

C. Tzourio

H. Chabriat

\title{
Frequency and Location of Dilated Virchow-Robin Spaces in Elderly People: A Population-Based 3D MR Imaging Study
}

BACKGROUND AND PURPOSE: dVRS have been previously associated with aging and cerebrovascular diseases. However, little is known about their prevalence and topographic distribution in the general elderly population.

MATERIALS AND METHODS: dVRS were evaluated by using high-resolution 3D MR imaging in 1826 subjects enrolled in the 3C-Dijon MR imaging study. On T1-weighted MR imaging, dVRS were detected according to 3D imaging criteria and rated by using 4-level severity scores based in the BG or in the WM. The number and anatomic location of large dVRS ( $\geq 3 \mathrm{~mm}$ ) were recorded.

RESULTS: dVRS were observed in the BG or WM in every subject. The severity of dVRS was significantly associated with higher age in both the BG and WM, whereas sex was related to the severity of dVRS only in the BG. Large dVRS were detected in $33.2 \%$ of participants. Status cribrosum was found in $1.3 \%$ of participants. dVRS were also highly prevalent within the hippocampus (44.5\%) and hypothalamus $(11.6 \%)$

CONCLUSIONS: dVRS are always detected in the BG or WM in elderly people, and large dVRS are also prevalent. The topographic distribution of dVRS is not uniform within the brain and may depend on anatomic or pathologic characteristics interacting with aging and sex.

ABBREVIATIONS: $\mathrm{BG}=$ basal ganglia; $3 \mathrm{C}=3$ city; $\mathrm{CADASIL}=$ cerebral autosomal dominant arteriopathy with subcortical infarcts and leukoencephalopathy; CMA = cortical medullary arteries; $\mathrm{dVRS}=$ dilated Virchow-Robin spaces; INSERM = French Institute of Health and Medical Research; LSA = lenticulostriate arteries; UMR = Mixed Unit of Research; WM = white matter

$\mathbf{V}$ irchow-Robin spaces are perivascular spaces that surround small vessels as they extend into the brain tissue. Virchow-Robin spaces can enlarge under undetermined conditions and become dVRS visualized on MR imaging in

Received July 31, 2010; accepted after revision September 8.

From INSERM U708 (Y.-C.Z., C.D., A.S., C.T., H.C.), Paris, France; Universités Pierre et Marie Curie-Paris 6 (Y.-C.Z., C.D., A.S., C.T.), Paris, France; Department of Neurology (Y.-C.Z., C.T., H.C.), Lariboisière Hospital, Paris, France; INSERM UMR 740 (Y.-C.Z., H.C.), Paris, France; Department of Neurology (Y.-C.Z.), Peking Union Medical College Hospital, Beijing, China; National Center for Scientific Research-French Atomic Energy Commission-UMR 6232 (B.M.), Groupe Imagerie Neurofonctionelle, Caen, France; Universités Caen Basse-Normandie (B.M.), Caen, France; Centre Hospitalier Universitaire de Caen (B.M.), Caen, France; Institut Universitaire de France (B.M.), Paris, France; and Department of Neuroradiology (F.R.), General Hospital, Dijon, France.

The 3C Study is conducted under a partnership agreement among INSERM, Victor Segalen-Bordeaux II University, and Sanofi-Aventis. The Fondation pour la Recherche Médicale funded the preparation and initiation of the study. The 3C Study is also supported by the Caisse Nationale Maladie des Travailleurs Salariés, Direction Générale de la Santé, Multi-Generator, Institut de la Longévité, Conseils Régionaux of Aquitaine and Bourgogne, Fondation de France, and the Ministry of Research-INSERM Programme "Cohortes et collections de données biologiques." Sponsors are involved neither in the design of the study nor in the data analyses or manuscript preparation.

Yi-Cheng Zhu is funded by the French Chinese Foundation for Science and Applications, China Scholarship Council, and Association de Recherche en Neurologie Vasculaire. Carole Dufouil has received consulting fees from Eisai pharmaceuticals. Christophe Tzourio has received investigator-initiated research funding from the French National Research Agency and has received fees from Sanofi-Synthelabo for participation in a data-safety monitoring board and from Merck-Sharp \& Dohm for participation in a scientific committee.

Please address correspondence to Hugues Chabriat, MD, Assistance Publique-Hôpitaux de Paris, Hôpital Lariboisière, Servicede Neurologie, 2, Rue Ambroise Paré, 75010 Paris, France; e-mail: hugues.chabriat@|rb.aphp.fr

Indicates open access to non-subscribers at www.ajnr.org

DOI 10.3174/ajnr.A2366 healthy subjects ${ }^{1}$ or in patients with stroke. ${ }^{2}$ Evidence is accumulating that dVRS are associated with WM lesions of vascular origin, ${ }^{3}$ dementia, ${ }^{1,4}$ or even multiple sclerosis. ${ }^{5}$ Although dVRS are widely detected in healthy individuals or in patients with stroke, their exact prevalence, severity, and topographic distribution in a large population of elderly subjects remains unknown and large epidemiologic studies of this MR imaging marker are still needed.

In previous MR imaging studies of dVRS, major technical limitations were encountered. First, most data were obtained with an MR imaging section thickness larger than $5 \mathrm{~mm}$, though the in-plane resolution could reach $1 \times 1 \mathrm{~mm}^{2}$ in some studies. ${ }^{1,2}$ Because dVRS are usually of $<2 \mathrm{~mm}$ in diameter in pathologic data and often present with a linear shape, ${ }^{6}$ the limited spatial MR imaging resolution obviously hinders the interpretation of their results. ${ }^{2}$ Second, almost all previous studies were based on the assessment of dVRS only in the axial planes, though 3D MR imaging analysis is of paramount importance to disclose the typical course of dVRS along the vascular trunk and for differential diagnosis with lacunar infarction. ${ }^{7}$

In the present study, we analyzed the prevalence, location, and severity of dVRS in a large community-based sample of elderly people. dVRS were identified and analyzed by using high-resolution 3D MR imaging and were graded by using dedicated severity scores both in the BG and within the WM.

\section{Materials and Methods}

\section{Subjects}

The 3C Study is a multicenter cohort study, conducted in 3 French cities (Bordeaux, Dijon, and Montpellier) and designed to estimate 
the risk of dementia attributable to vascular risk factors. To be enrolled, subjects had to be $\geq 65$ years of age, noninstitutionalized, and registered on electoral rolls. The detailed description of the study protocol has been previously reported. ${ }^{8}$ The study was approved by the ethics committee of the University Hospital of Kremlin-Bicêtre. Each participant signed an informed consent.

The participants from Dijon who were younger than 80 years of age and enrolled between June 1999 and September $2000(n=2763)$ were scheduled to have a cerebral MR imaging examination. Exclusion criteria for the examination were the following: cardiac pacemaker; valvular prosthesis, other internal electrical/magnetic devices; history of neurosurgery/aneurysm; claustrophobia; and the presence of metal fragments (eyes, brain, and spinal cord). Although 2285 subjects $(82.7 \%)$ agreed to participate, only 1924 subjects underwent brain MR imaging because of financial limitations, among whom 1876 were available for the current analysis. ${ }^{9}$

Individuals with dementia, brain tumors, and self-reported history of stroke were excluded from the current analysis $(n=58)$, and the final sample size comprised 1818 subjects.

\section{MR Imaging Data}

MR imaging was performed on a 1.5T Magnetom scanner (Siemens, Erlangen, Germany). A 3D high-resolution T1-weighted brain volume was acquired by using a 3D inversion-recovery fast-spoiled gradient-echo sequence $(\mathrm{TR}=97 \mathrm{~ms}$; $\mathrm{TE}=4 \mathrm{~ms}$; $\mathrm{TI}=600 \mathrm{~ms}$; coronal acquisition). The axially reoriented $3 \mathrm{D}$ volume matrix size was $256 \times 192 \times 256$ with a $1.0 \times 0.98 \times 0.98 \mathrm{~mm}^{3}$ voxel size. There were 124 sections covering the whole brain. T2- and proton attenuationweighted brain volumes were acquired by using a $2 \mathrm{D}$ dual spin-echo sequence with $2 \mathrm{TEs}(\mathrm{TR}=4400 \mathrm{~ms} ; \mathrm{TE} 1=16 \mathrm{~ms}$; TE2 $=98 \mathrm{~ms})$. T2 and proton-attenuation acquisitions consisted of 35 axial sections 3.5-mm thick (0.5-mm between-section spacing) having a $256 \times 256$ matrix size and a $0.98 \times 0.98 \mathrm{~mm}^{2}$ in-plane resolution.

\section{D MR Imaging Analysis}

Image analysis was performed on MRIcro 1.40 (http://www.sph. sc.edu/comd/rorden/mricro.html), a free image-processing program operating on a Microsoft Windows platform. For each subject's scan, the first step of MR imaging analysis was performed with T1-weighted images at $\times 2$ magnification on a 27 -inch screen to visualize the characteristics of lesions simultaneously in axial, coronal, and sagittal planes. Thus, all suspected lesions were analyzed on the basis of their shape and location in the 3D space (Fig 1). T2- and proton attenuation-weighted images were analyzed in a second step to confirm that the signal intensity of the lesion corresponded to that of CSF.

\section{Rating of dVRS}

dVRS were defined as CSF-like signal-intensity (hypointense on T1 and hyperintense on T2) lesions that were round, ovoid, or linear; $<3$ $\mathrm{mm}$ in their maximum diameter, with smooth delineated contours; and located in areas supplied by perforating arteries (Fig 2). For lesions fulfilling the same criteria except that their diameter was $\geq 3$ $\mathrm{mm}$, further effort was needed to differentiate them from infarcts by using multiplanar reformatting. Only those with a typical vascular shape and following the orientation of perforating vessels (including cystic lesions with an extension of vascular shape) were then regarded as dVRS (Fig 1). ${ }^{7}$

For each subject, all 124 axially oriented T1-weighted sections were first examined to evaluate the global burden of dVRS and to identify the section containing the largest number of dVRS in both the
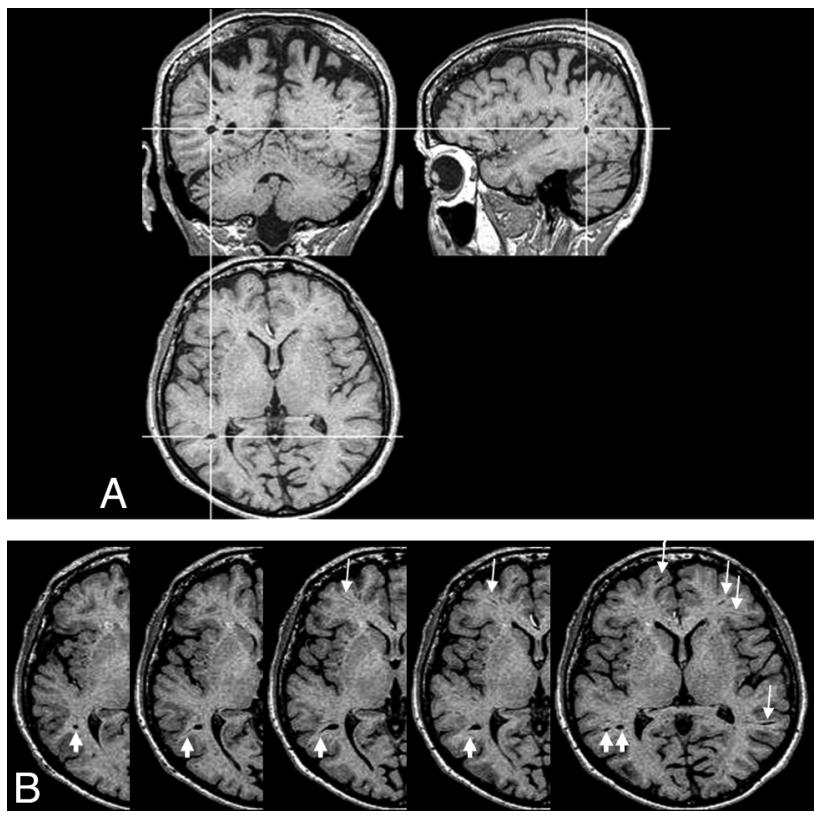

Fig 1. 3D T1-weighted images from 1 participant showing multiple dVRS. $A$, Cystiform dVRS are analyzed on axial, coronal, and sagittal planes. $B$, Multiple dVRS exhibit a linear shape and orientation along cortical medullary arteries (thin white arrows) in the white matter. Cystic dVRS (large white arrows) are detected on contiguous sections showing a cavity with a vascular shape extension.

BG and cerebral WM. In BG, dVRS were then rated according to a 4-level severity score in the section containing the greatest number of dVRS. The degrees of dVRS were defined as follows: degree 1, when there were $<5 \mathrm{dVRS}$; degree 2 , when there were between 5 and 10 dVRS; degree 3 , when there were $>10 \mathrm{dVRS}$ but they were still countable; and degree 4 , when an innumerable number of dVRS resulted in a cribriform change in the BG (Fig 3). In cerebral WM, dVRS were scored as follows: degree 1 , when there were $<10$ dVRS in the total WM; degree 2, when there were $>10$ dVRS in the total WM but $<10$ in the section containing the greatest number of dVRS; degree 3 , when there were between 10 and 20 dVRS in the section containing the greatest number of dVRS; and degree 4 , when there were $>20 \mathrm{dVRS}$ in the section containing the greatest number of dVRS (Fig 4). This rating scheme was adopted after testing different visual rating methods, including those reported in the literature on a subset of MR imaging data from the first 150 subjects of the cohort and after performing step-by-step multiple rectifications. dVRS with axial diameters of $\geq 3 \mathrm{~mm}$ were defined as "large dVRS" in our study. Their presence, number, and location were systematically recorded.

All images were analyzed by the same experienced reader (Y.-C.Z.) who was blinded to all clinical data. When this reader was uncertain of the categorization of MR imaging signal-intensity abnormalities $(n=113)$, the corresponding lesions were reviewed by 2 other readers who had extensive experience in MR imaging studies (H.C. and C.T.) and a decision was made on the basis of the majority vote of the 3 readers.

\section{Other MR Imaging Parameters}

The gray matter, WM, and CSF volumes were estimated with voxelbased morphometry methods detailed elsewhere. ${ }^{10}$ Total intracranial volume was computed as the sum of the gray matter, WM, and CSF volumes and brain parenchymal fraction as the ratio of brain tissue volume to total intracranial volume. 

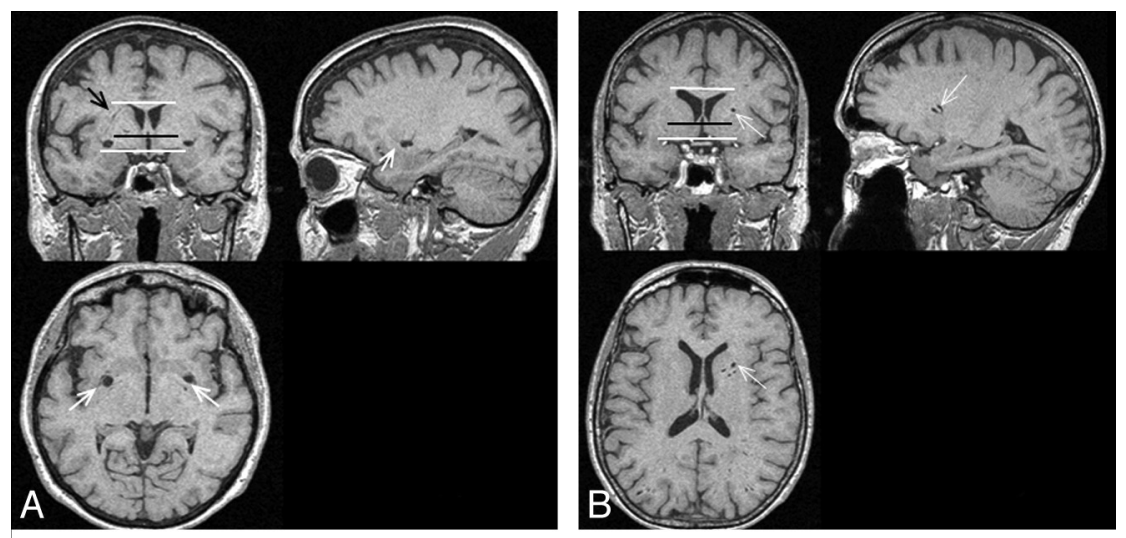

Fig 2. Locations of dVRS. A, In coronal sections, the upper white line crosses the superior limit of the caudate on both sides; the lower one crosses the base of the forebrain at the level of the anterior perforated substance. The lower onethird of the section between these 2 lines (between the black line and lower white line) is defined as belonging to the proximal part of the LSA, whereas the upper two-thirds (between the black line and upper white line) is considered as the distal part of the LSA. Large dVRS are detected in the proximal part of the LSA (white arrows) on both sides; a linear dilated Virchow-Robin space is also detected in the distal part of the LSA (black arrow). B, Large dVRS (diameter of $3 \mathrm{~mm}$ ) are detected in the left distal part of the LSA (white arrows). C, Two Virchow-Robin spaces are seen in the left side of the hippocampus (arrows). D, A Virchow-Robin space is seen in the in right hypothalamus (arrows), as a small dot in the axial section and as a vascular shape in the longitu-
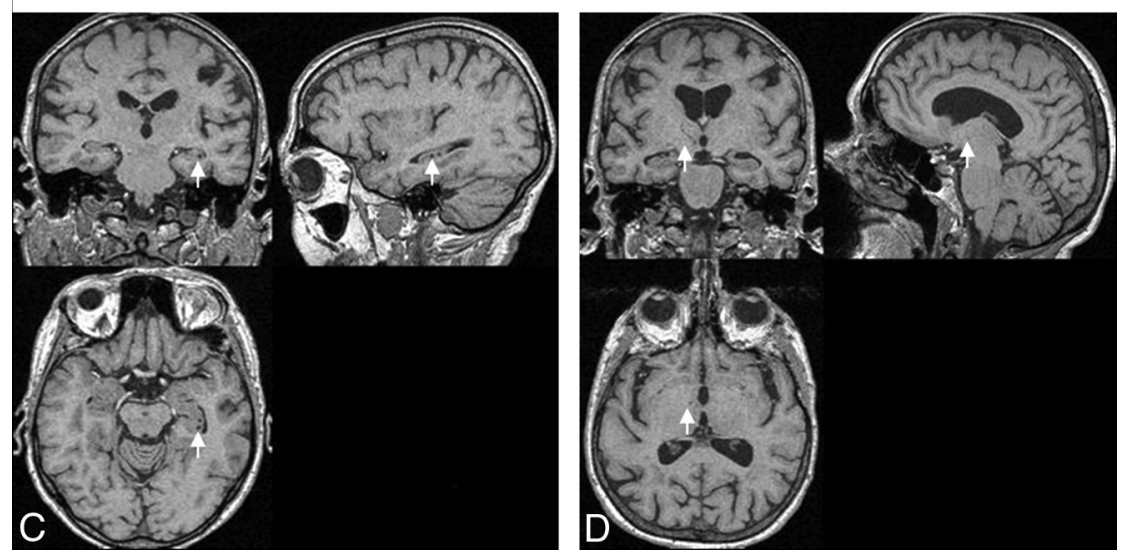
dinal section
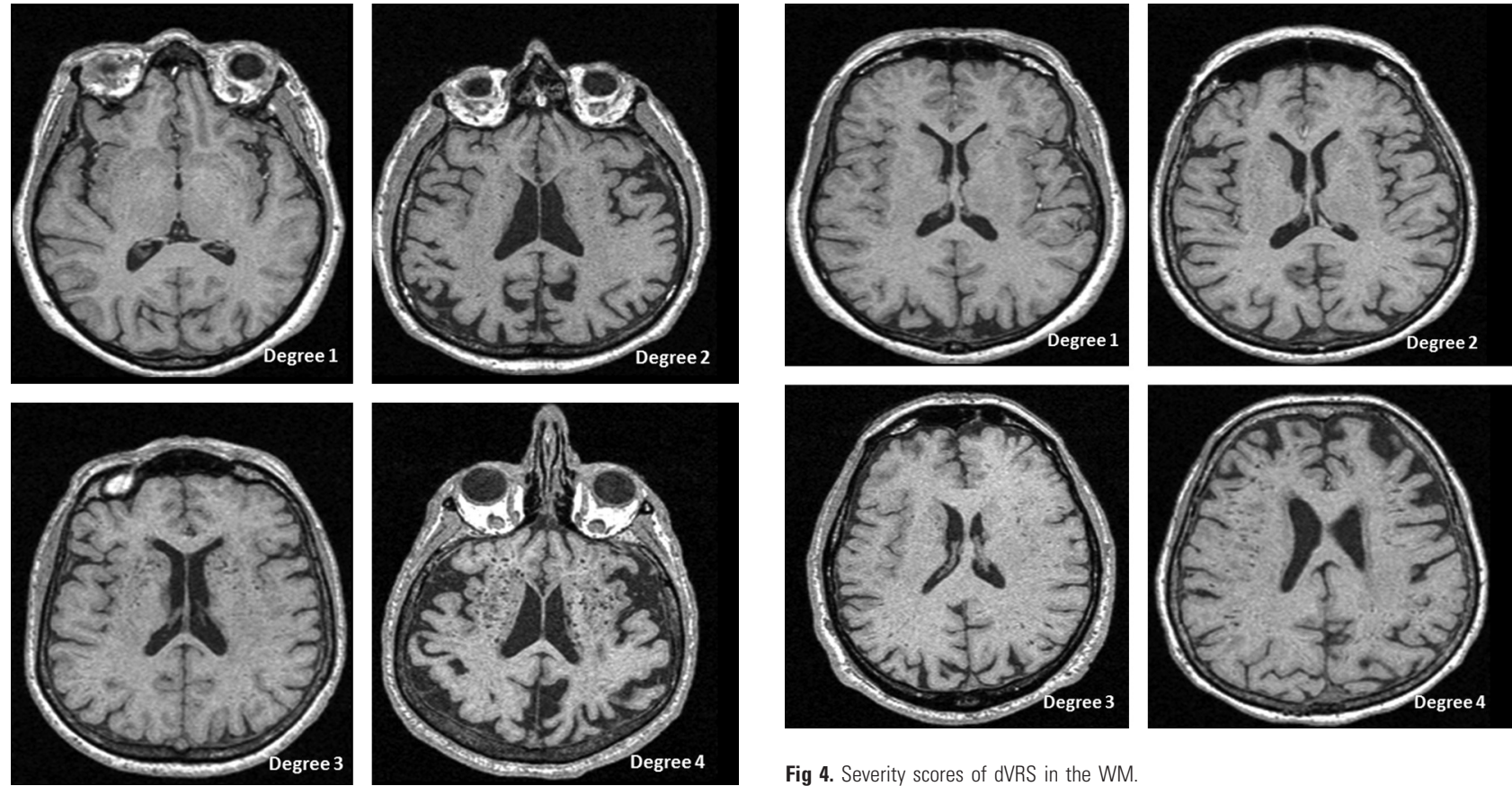

Fig 4. Severity scores of dVRS in the WM.

Fig 3. Severity scores of dVRS in the BG.

\section{Statistical Analysis}

Associations between qualitative variables and dVRS scores in the BG or WM were assessed by using $\chi^{2}$ tests. In multivariable analyses, the associations between qualitative variables and $\mathrm{dVRS}$ degree in the BG or WM were assessed by using logistic regression controlling for brain size (estimated by total intracranial volume). Associations between continuous variables and dVRS degrees in the BG or WM were exam-

ined by using analysis of covariance controlling for total intracranial volume. Potential interactions were tested by adding cross-products into the models. All analyses were performed by using SAS, Version 9.1 (SAS Institute, Cary, North Carolina).

\section{Results}

The sample comprised 1818 participants, $61.2 \%$ of whom were women. The mean age was $72.5 \pm 4.1$ years. The intrarater agreement for the rating of dVRS in the BG and WM was 


\begin{tabular}{|c|c|c|c|c|c|}
\hline \multicolumn{6}{|c|}{$\begin{array}{l}\text { Table 1: Cross table of dVRS scores in basal ganglia and white } \\
\text { matter }\end{array}$} \\
\hline \multirow[b]{2}{*}{$\begin{array}{l}\text { Scores of } \\
\text { dVRS in } B G\end{array}$} & \multicolumn{4}{|c|}{ Scores of dVRS in WM, No. (\%) } & \multirow[b]{2}{*}{ Total No. $(\%)$} \\
\hline & $\begin{array}{c}\text { Degree } \\
1\end{array}$ & $\begin{array}{c}\text { Degree } \\
2\end{array}$ & $\begin{array}{c}\text { Degree } \\
3\end{array}$ & $\begin{array}{c}\text { Degree } \\
4\end{array}$ & \\
\hline Degree 1 & $295(16.2)$ & $528(29.0)$ & $127(7.0)$ & $26(1.4)$ & $976(53.7)$ \\
\hline Degree 2 & $104(5.7)$ & 344 (18.9) & $147(8.1)$ & $46(2.5)$ & $641(35.3)$ \\
\hline Degree 3 & $26(1.4)$ & $88(4.8)$ & $45(2.5)$ & $18(1.0)$ & $177(9.7)$ \\
\hline Degree 4 & $4(0.2)$ & $10(0.6)$ & $6(0.3)$ & $4(0.2)$ & $24(1.3)$ \\
\hline Total & $429(23.6)$ & $970(53.4)$ & $325(17.9)$ & $94(5.2)$ & $1818(100 \%)$ \\
\hline
\end{tabular}

\begin{tabular}{|c|c|c|c|c|c|}
\hline \multirow[b]{2}{*}{ Location } & \multicolumn{5}{|c|}{ Scores of dVRS } \\
\hline & No. & $\begin{array}{l}\text { Mean age } \\
\text { (yr) (SD) }\end{array}$ & $P$ Value & $\begin{array}{c}\% \text { Men } \\
\text { (No.) }\end{array}$ & $P$ Value \\
\hline \multicolumn{6}{|l|}{$\overline{\mathrm{BG}}$} \\
\hline Degree 1 & 976 & $71.7(3.9)$ & $<.0001^{\mathrm{a}} /<.0001^{\mathrm{b}}$ & 36.5 (356) & $.0002^{c} / .002^{d}$ \\
\hline Degree 2 & 641 & $73.0(4.2)$ & & $37.8(242)$ & \\
\hline Degree 3 & 177 & $74.2(4.0)$ & & 50.3 (89) & \\
\hline Degree 4 & 24 & $75.0(3.4)$ & & $79.2(19)$ & \\
\hline \multicolumn{6}{|l|}{ WM } \\
\hline Degree 1 & 429 & $72.2(4.2)$ & $.004^{a} / .004^{b}$ & $33.6(144)$ & $.51^{c} / .53^{d}$ \\
\hline Degree 2 & 970 & $72.4(4.1)$ & & $39.5(383)$ & \\
\hline Degree 3 & 325 & $72.7(4.2)$ & & $42.2(137)$ & \\
\hline Degree 4 & 94 & $73.8(4.1)$ & & $44.7(42)$ & \\
\hline
\end{tabular}

${ }^{a}$ Computed from analysis of covariance controlling for total intracranial volume. b Computed from analysis of covariance controlling for total intracranial volume and sex. c Computed from logistic regression controlling for total intracranial volume.

${ }^{d}$ Computed from logistic regression controlling for total intracranial volume and age.

assessed on a random sample of 100 individuals at 1-month intervals starting from the first reading. The $\kappa$ statistics reached values of 0.77 and 0.75 , respectively, indicating a good reliability.

dVRS were detected in every subject. Most participants (89\%) had dVRS with scores of 1 or 2 in the BG. Degree 4 in the BG was observed in only 24 (1.3\%) individuals. Within the WM, $77 \%$ of the individuals had dVRS scores of 1 or 2 ; only 94 subjects $(5.2 \%)$ had the highest degree.

The scores of dVRS in the BG were positively associated with those obtained in the WM $\left(\chi^{2}=100,9 d f, P<.0001\right)$ (Table 1). However, a discrepancy of $\geq 2^{\circ}$ between the WM and BG scores of dVRS was observed in 239 individuals $(13.1 \%)$ (Table 1$)$. Mean age increased with the severity of dVRS both in the BG $(P<.0001$, Table 2$)$ and WM $(P=.004$, Table 2$)$. These associations were not modified by sex ( $P$ for interaction, $\leq 0.51$ for all tests; Table 2$)$. The proportion of men increased with the severity of dVRS in the BG $(P=.0002)$ but not in the WM $(P=.51)$. There were no significant interactions between age and sex on the severity of dVRS ( $P$ for interaction, $\leq 0.40$ for all tests). Additional adjustments of the models on age did not alter the results (Table 2).

dVRS were frequently detected in the hippocampus (frequency, $44.5 \%$ ) and hypothalamus (frequency, 11.6\%). These dVRS were found to have a uniform location and shape across subjects (Fig 2). Among subjects with dVRS in the hippocampus, dVRS were found bilaterally in $48.8 \%$ of subjects, and among subjects with dVRS in the hypothalamus, they were found bilaterally in $45.7 \%$ of subjects.

Overall, large dVRS were detected in 603 (33.2\%) subjects; 388 subjects $(21.3 \%)$ had only one, $139(7.7 \%)$ had 2 , and 76
(4.2\%) had $>2$ large dVRS (there were 948 large dVRS in total). Large dVRS were observed in the following areas by decreasing frequency: proximal LSA, 19.9\%; CMA, 10.2\%, hippocampus, $5.8 \%$, distal LSA, $2.8 \%$, brain stem, $1.2 \%$; and hypothalamus, $0.1 \%$. Therefore, half of the large dVRS were detected in the proximal part of LSA, and one-fourth along the CMA. The prevalence of large dVRS in the proximal part of LSA, in the distal part of LSA, or along CMA significantly increased with the severity of dVRS in the BG and WM $(P \leq$ .02 for all tests).

\section{Discussion}

In this sample of 1818 elderly subjects free of dementia or stroke, we found that dVRS were a common MR imaging finding. Using high-resolution 3D MR imaging, we found dVRS in every subject and dVRS of $>3 \mathrm{~mm}$ in approximately one-third of them. The results also showed that the number of dVRS increased with age in the BG as well as in the WM and that men had more dVRS in the BG than women.

The observation of dVRS in all subjects of this cohort contrasts with the $38 \%$ prevalence reported in MR imaging studies of 816 outpatients of wide age range and $3 \%$ of 1250 pediatric patients. ${ }^{2,11}$ The different spatial resolutions of imaging may be the main source of this discrepancy. Herein, we used 3D MR imaging acquisitions with a voxel size of $1.0 \times 0.98 \times 0.98 \mathrm{~mm}^{3}$, whereas most previous studies of dVRS including the above-mentioned were based on 2D MR imaging acquisitions and image sections of 5-mm thickness. ${ }^{1,2,11,12}$ Moreover, Groeschel et $\mathrm{al}^{7}$ found a higher detectability of dVRS on coronal T1-weighted images than on axial T2, sagittal T2, or axial fluid-attenuated inversion recovery images. ${ }^{7}$ These data emphasized the importance of section orientation and MR imaging sequences in the assessment of dVRS. In the present study, the 3D T1 imaging analysis used for identification of dVRS may have also increased the detection of dVRS. Virchow-Robin spaces were also visible in $100 \%$ of subjects in another study using high-resolution 3D MR imaging in 125 healthy subjects between 1 and 30 years of age, supporting our inferences. ${ }^{7}$

Although a diameter of $3 \mathrm{~mm}$ has been widely used as a cutoff value to discriminate silent brain infarcts from dilated perivascular spaces on MR imaging, ${ }^{13} \mathrm{dVRS}$ with diameters of $>3 \mathrm{~mm}$ are frequently detected. ${ }^{14}$ One-third of individuals in the 3C-Dijon MR imaging sample had 1 or more dVRS larger than $3 \mathrm{~mm}$. This is 3 times higher than the prevalence of lacunar infarctions in the same sample. ${ }^{9}$ The most common location of large dVRS was found to be at the proximal part of LSA, in agreement with the most widely recognized location of dilated perivascular spaces. ${ }^{6,14,15}$ The prevalence of large dVRS was twice as high in this area as along the CMA and 10 times higher than in the distal part of LSA. Various mechanisms underlying the expansion of perivascular spaces have been previously suggested, such as the following: 1) changes in the permeability of the arterial wall, ${ }^{16} 2$ ) altered drainage and accumulation of amyloid proteins along the vessels, ${ }^{17} 3$ ) spiral elongation of blood vessels, ${ }^{15}$ or 4 ) cerebral atrophy. ${ }^{18}$ The higher frequency of large dVRS at the proximal part of LSA may be particularly related to additional anatomic characteristics, including the angular trajectory of the vascular tree and the presence of multiple branches crossing the surface of the cerebrum in this area. 
In this study, an accumulation of uncountable dVRS in the BG was observed in 1.3\% of participants. A special MR imaging change of cribriform cavities in the BG was the feature of this group. An "état criblé" or "status cribrosum" has been previously proved to be multiple dVRS in pathologic studies ${ }^{19}$ and has been reported only among cases of vascular Parkinsonism, ${ }^{20}$ subcortical ischemic vascular dementia, ${ }^{21,22}$ or CADASIL ${ }^{23}$ but has not been reported in the general population, to our knowledge. The present data further emphasize that the accumulation of multiple dVRS is not rare and can occur in elderly people in the absence of severe disability or dementia.

The association between age and the severity of dVRS was consistently detected in previous dVRS studies in hospitalized patients ${ }^{2}$ and in patients with lacunar infarction, ${ }^{12}$ as well as in specific conditions such as CADASIL. ${ }^{23}$ This may suggest 1 or several potential causes of dVRS with aging. ${ }^{15}$ In this study, a higher grade of dVRS in the BG was observed more frequently in men than in women. This difference was independent of the total intracranial volume. Most interesting, this sex effect was not detected for dVRS in the WM. Whether variations in ultrastructural characteristics or permeability properties of vessels located within the BG are involved in the male sex association with higher severity of dVRS in the BG will require specific investigation.

In this sample, we also observed that dVRS are commonly detected in areas not usually considered for MR imaging assessment of dilated perivascular spaces. Although still controversial, the hippocampal cavities located between the cornu ammonis and dentate gyrus were found at postmortem examination to be covered by a single cell layer, to contain blood vessels, and to be surrounded by intact cerebral parenchyma, which strongly suggest typical dVRS. ${ }^{24}$ We found that these cavities are of uniform shape and location, with a frequency of $44.5 \%$ in the 3C-Dijon MR imaging cohort, close to the 39\% reported by Sasaki et $\mathrm{al}^{24}$ in 109 patients from 8 to 85 years of age. Whether their severity is related to age and apolipoprotein E4 or E2 alleles as suggested in small samples will need additional investigations. ${ }^{25,26} \mathrm{We}$ also found that $>10 \%$ of patients presented with dVRS in the hypothalamus, a feature never previously reported, to our knowledge.

The strengths of this study include the population-based design, the large sample size, and the use of high-resolution MR imaging and 3D image analysis. The definition of dVRS was also based on strict anatomic and imaging criteria, and the method of assessment proved to be highly reliable. The main limitations include the potential misclassification of dVRS versus small ischemic lesions despite the training of the reader in using $3 \mathrm{D}$ imaging analysis and the use of a semiquantitative evaluation for grading of severity.

\section{Conclusions}

The results of this study emphasize that large dVRS are highly prevalent in the healthy population and can be detected in most brain structures by using 3D high-resolution MR imaging. The results confirmed that the topographic distribution of dVRS is not uniform within the brain and may depend on anatomic or pathologic characteristics interacting with aging and sex. Whether the severity of dVRS at MR imaging is related to vascular risk factors and/or to other imaging markers of small vessel diseases in healthy elderly people will need additional investigation.

\section{Acknowledgments}

We thank Denis Krause, MD, from the Departments of Radiology and Medical Imaging, Bocage Hospital, Dijon, France, for his work in MR imaging data collection.

\section{References}

1. Maclullich AM, Wardlaw JM, Ferguson KJ, et al. Enlarged perivascular spaces are associated with cognitive function in healthy elderly men. J Neurol Neurosurg Psychiatry 2004;75:1519-23

2. Heier LA, Bauer CJ, Schwartz L, et al. Large Virchow-Robin spaces: MR-clinical correlation. AJNR Am J Neuroradiol 1989;10:929-36

3. Udaka F, Sawada H, Kameyama M. White matter lesions and dementia: MRIpathological correlation. Ann N Y Acad Sci 2002;977:411-15

4. Patankar TF, Mitra D, Varma A, et al. Dilatation of the Virchow-Robin space is a sensitive indicator of cerebral microvascular disease: study in elderly patients with dementia. AJNR Am J Neuroradiol 2005;26:1512-20

5. Wuerfel J, Haertle M, Waiczies H, et al. Perivascular spaces: MRI marker of inflammatory activity in the brain? Brain 2008;131:2332-40

6. Bokura H, Kobayashi S, Yamaguchi S. Distinguishing silent lacunar infarction from enlarged Virchow-Robin spaces: a magnetic resonance imaging and pathological study. J Neurol 1998;245:116-22

7. Groeschel S, Chong WK, Surtees R, et al. Virchow-Robin spaces on magnetic resonance images: normative data, their dilatation, and a review of the literature. Neuroradiology 2006;48:745-54

8. Alperovitch A. Vascular factors and risk of dementia: design of the three-city study and baseline characteristics of the study population. Neuroepidemiology 2003;22:316-25

9. Soumare A, Elbaz A, Zhu Y, et al. White matter lesions volume and motor performances in the elderly. Ann Neurol 2009;65:706-15

10. Lemaitre H, Crivello F, Grassiot B, et al. Age- and sex-related effects on the neuroanatomy of healthy elderly. Neuroimage 2005;26:900-11

11. Rollins NK, Deline C, Morriss MC. Prevalence and clinical significance of dilated Virchow-Robin spaces in childhood. Radiology 1993;189:53-57

12. Rouhl RP, van Oostenbrugge RJ, Knottnerus IL, et al. Virchow-Robin spaces relate to cerebral small vessel disease severity. J Neurol 2008;255:692-96

13. Vermeer SE, Den HT, Koudstaal PJ, et al. Incidence and risk factors of silent brain infarcts in the population-based Rotterdam Scan Study. Stroke 2003;34:392-96

14. Takao M, Koto A, Tanahashi N, et al. Pathologic findings of silent, small hyperintense foci in the basal ganglia and thalamus on MRI. Neurology 1999;52:666-68

15. Kwee RM, Kwee TC. Virchow-Robin spaces at MR imaging. Radiographics 2007;27:1071-86

16. Pollock H, Hutchings $M$, Weller RO, et al. Perivascular spaces in the basal ganglia of the human brain: their relationship to lacunes. J Anat 1997;191(pt 3):337-46

17. Roher AE, Kuo YM, Esh C, et al. Cortical and leptomeningeal cerebrovascular amyloid and white matter pathology in Alzheimer's disease. Mol Med 2003;9:112-22

18. van Swieten JC, van den Hout JH, van Ketel BA, et al. Periventricular lesions in the white matter on magnetic resonance imaging in the elderly: a morphometric correlation with arteriolosclerosis and dilated perivascular spaces. Brain 1991;114(pt 2):761-74

19. Mancardi GL. Neuropathologic study of lacunae and cribriform cavities of the brain. Eur Neurol 1989;29(suppl 2):16-19

20. Fenelon G, Gray F, Wallays C, et al. Parkinsonism and dilatation of the perivascular spaces (état criblé) of the striatum: a clinical, magnetic resonance imaging, and pathological study. Mov Disord 1995;10:754-60

21. Braffman BH, Zimmerman RA, Trojanowski JQ, et al. Brain MR: pathologic correlation with gross and histopathology. 1. Lacunar infarction and Virchow-Robin spaces. AJR Am J Roentgenol 1988;151:551-58

22. Roman GC. On the history of lacunes, état criblé, and the white matter lesions of vascular dementia. Cerebrovasc Dis 2002;13(suppl 2):1-6

23. Cumurciuc R, Guichard JP, Reizine D, et al. Dilation of Virchow-Robin spaces in CADASIL. Eur J Neurol 2006;13:187-90

24. Sasaki M, Sone M, Ehara S, et al. Hippocampal sulcus remnant: potential cause of change in signal intensity in the hippocampus. Radiology 1993;188:743-46

25. Barboriak DP, Doraiswamy PM, Krishnan KR, et al. Hippocampal sulcal cavities on MRI: relationship to age and apolipoprotein E genotype. Neurology 2000;54:2150-53

26. Bastos-Leite AJ, van Waesberghe JH, Oen AL, et al. Hippocampal sulcus width and cavities: comparison between patients with Alzheimer disease and nondemented elderly subjects. AJNR Am J Neuroradiol 2006;27:2141-45 